\title{
Development of Global Cortical Networks in Early Infancy
}

\author{
Fumitaka Homae, ${ }^{1}$ Hama Watanabe, ${ }^{2}$ Takayuki Otobe, ${ }^{3}$ Tamami Nakano, ${ }^{4,5}$ Tohshin Go, ${ }^{6,7}$ Yukuo Konishi, ${ }^{6,7}$ \\ and Gentaro Taga ${ }^{2,8}$ \\ ${ }^{1}$ Department of Language Sciences, Tokyo Metropolitan University, Hachioji-shi, Tokyo 192-0397, Japan, ${ }^{2}$ Graduate School of Education, University of \\ Tokyo, Bunkyo-ku, Tokyo 113-0033, Japan, ${ }^{3}$ Department of Early Childhood Education, Jin-ai Women's College, Fukui-shi, Fukui 910-0124, Japan, \\ ${ }^{4}$ Department of Neurophysiology, Juntendo University, Bunkyo-ku, Tokyo 113-8421, Japan, ${ }^{5}$ Japan Society for the Promotion of Science, ${ }^{6}$ Center for Baby \\ Science, Doshisha University, Kizugawa-shi, Kyoto 619-0225, Japan, ${ }^{7}$ Tokyo Women's Medical University, Shinjuku-ku, Tokyo 162-8666, Japan, and ${ }^{8}$ Core \\ Research for Evolutional Science and Technology, Japan Science and Technology Agency, Kawaguchi-shi, Saitama 332-0012, Japan
}

Human cognition and behaviors are subserved by global networks of neural mechanisms. Although the organization of the brain is a subject of interest, the process of development of global cortical networks in early infancy has not yet been clarified. In the present study, we explored developmental changes in these networks from several days to 6 months after birth by examining spontaneous fluctuations in brain activity, using multichannel near-infrared spectroscopy. We set up 94 measurement channels over the frontal, temporal, parietal, and occipital regions of the infant brain. The obtained signals showed complex time-series properties, which were characterized as $1 / f$ fluctuations. To reveal the functional connectivity of the cortical networks, we calculated the temporal correlations of continuous signals between all the pairs of measurement channels. We found that the cortical network organization showed regional dependency and dynamic changes in the course of development. In the temporal, parietal, and occipital regions, connectivity increased between homologous regions in the two hemispheres and within hemispheres; in the frontal regions, it decreased progressively. Frontoposterior connectivity changed to a "U-shaped" pattern within 6 months: it decreases from the neonatal period to the age of 3 months and increases from the age of 3 months to the age of 6 months. We applied cluster analyses to the correlation coefficients and showed that the bilateral organization of the networks begins to emerge during the first 3 months of life. Our findings suggest that these developing networks, which form multiple clusters, are precursors of the functional cerebral architecture.

\section{Introduction}

The structural and functional development of the brain during infancy has been a subject of interest since early times (Johnson, 2005). The infant brain is not a miniature version of the adult brain but a continuously self-organizing system that forms functional regions and networks among multiple regions via short-range and long-range connectivity. Sensory, motor, and cognitive functions in infancy show changes associated with the development of this system. Although recent neuroimaging studies successfully revealed functional localization in the infant brain (Dehaene-Lambertz et al., 2002; Peña et al., 2003; Taga et al., 2003; Homae et al., 2006), it has not been clarified how multiple functional regions and their global networks emerge during the course of early development.

The organization of brain networks in early infancy on the basis of structural development is an extensively debated issue. One viewpoint is that the connectivity between cortical regions gradually increases. During the first 6 months, increases in the synaptic density occur in the sensory and association regions (Huttenlocher, 2002). The progression of myelination in the

Received Nov. 13, 2009; revised Feb. 22, 2010; accepted Feb. 26, 2010.

This work was partly supported by Grants-in-Aid for Scientific Research 21613006 (F.H.) and 20670001 (G.T.). We thank Kayo Asakawa, Masako Ito, and Kyoko Ono for their administrative and technical assistance.

Correspondence should be addressed to Fumitaka Homae, Department of Language Sciences, Tokyo Metropolitan University, 1-1 Minami Osawa, Hachioji-shi, Tokyo 192-0397, Japan. E-mail: fhomae@tmu.ac.jp.

DOI:10.1523/JNEUROSCI.5618-09.2010

Copyright $\odot 2010$ the authors $\quad 0270-6474 / 10 / 304877-06 \$ 15.00 / 0$ postnatal period has been documented in magnetic resonance imaging (MRI) (Paus et al., 2001) and diffusion tensor MRI studies (Hermoye et al., 2006; Dubois et al., 2008). Another viewpoint is that excessive connections are present among cortical regions, and the number of connections decreases after birth so that the remaining networks function effectively. Postnatal decrease in the number of callosal axons has been observed in the developing rhesus monkey (LaMantia and Rakic, 1990). Studies on the structural development of the brain have raised the critical question of how functional networks develop in infancy. In the present study, we report our novel findings of dynamic changes in the functional connectivity of the human brain; these changes reflect both increases and decreases in structural connectivity.

Functional networks in adults have been studied by examining the patterns of spontaneous fluctuations in brain activity (Biswal et al., 1995; Salvador et al., 2005; Buckner and Vincent, 2007; Fox and Raichle, 2007; Seeley et al., 2009; White et al., 2009). Spontaneous fluctuations have been reported in the cerebral blood oxygenation in sleeping infants (Taga et al., 2000). Spontaneous fluctuations were measured during the task-free (resting) state; this method is especially useful for investigating neural systems at multiple stages of development. In the present study, we examined the development of cortical networks in the infant brain by using multichannel near-infrared spectroscopy (NIRS) (Jöbsis, 1977; Villringer and Chance, 1997). Developmental changes in the patterns of the spontaneous fluctuations would affect brain organization, 
A

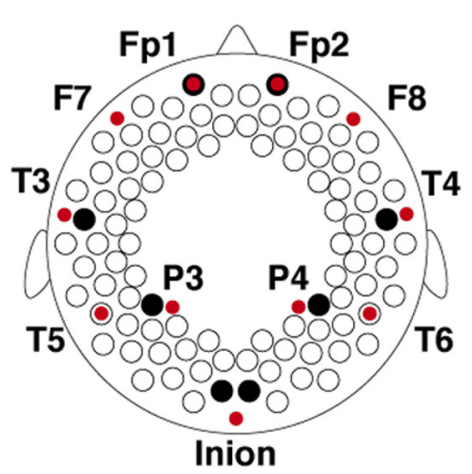

C

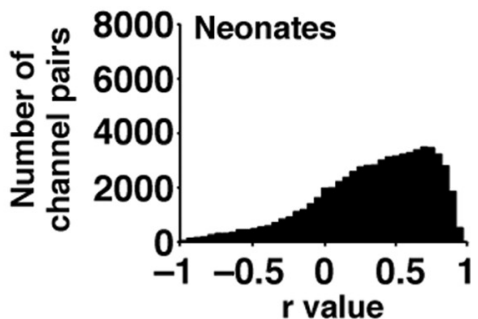

D

Drontal
Fp1, Fp2
Tempora

T3, T4

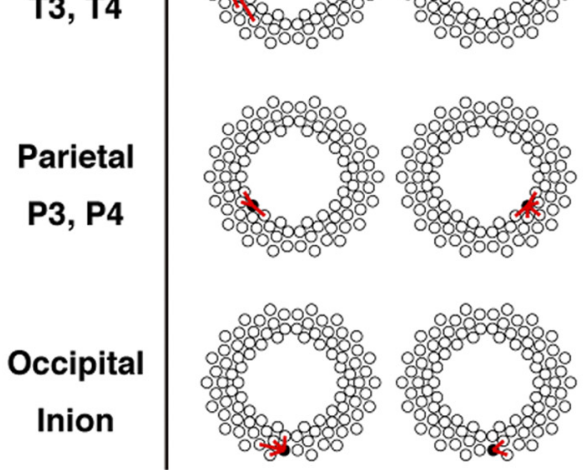

B
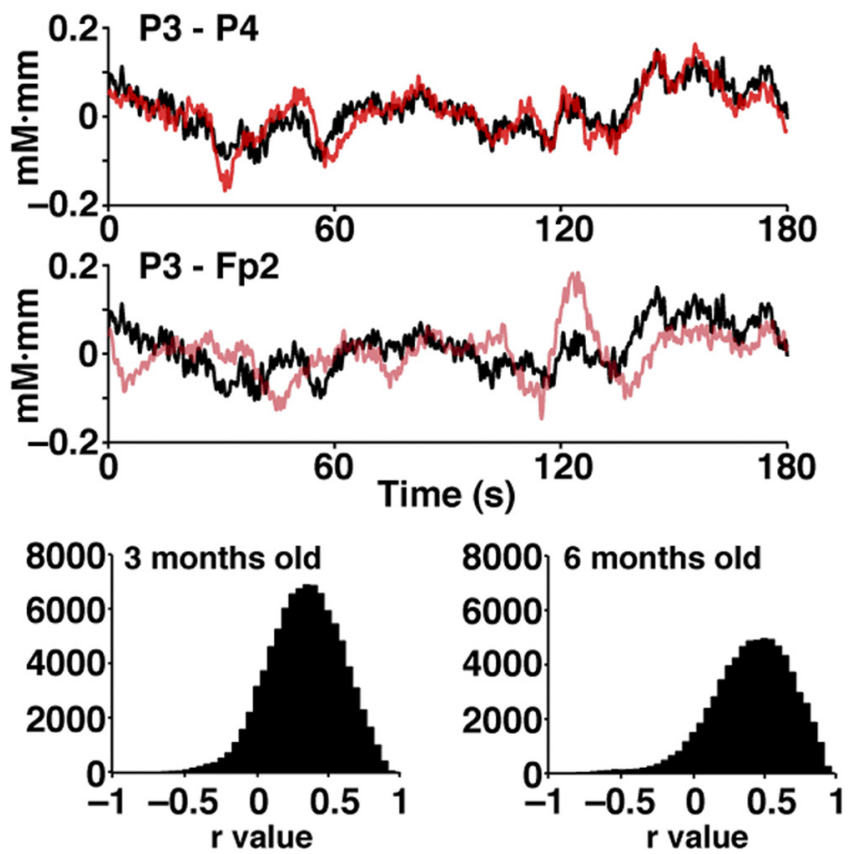

3 months old

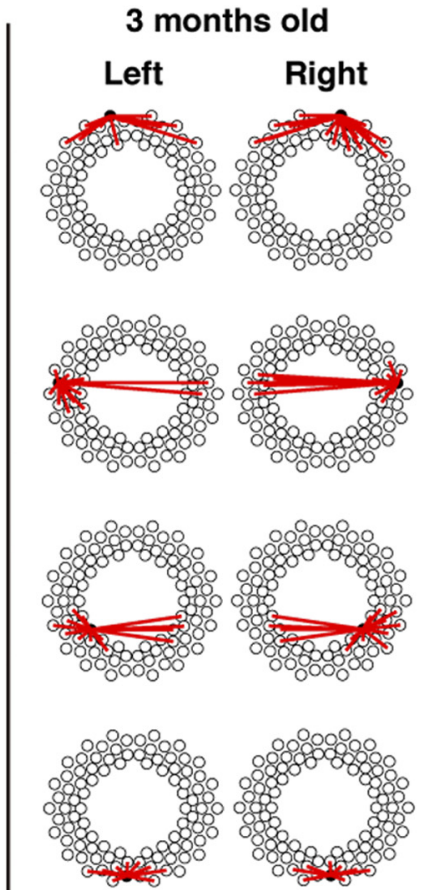

Figure 1. Functional networks characterized by spontaneous fluctuations in brain activity in the infants. $A$, Probe settings of the 94 -channel NIRS (sampling rate, $10 \mathrm{~Hz}$ ). Several landmarks from the international $10-20$ system are shown (red dots). Filled circles indicate the channels shown in $\boldsymbol{D}$. $\boldsymbol{B}$, Representative examples of continuous data of a 6 -month-old infant. The black and red lines in the top show the data of the measurement channel near P3 and P4, respectively. This pair has a high temporal correlation. In the bottom, the black and red lines show the data of P3 and Fp2, respectively. The correlation between the two channels was lower than that between P3 and P4. C, The distribution of correlation coefficients ( $r$ values) calculated using 3 min continuous data. The ordinates denote the total number of channel pairs across the infants. $\boldsymbol{D}$, Representative correlations. The red lines show correlations that were higher than 0.5 (averaged across all infants in each group). See also supplemental Figures $2-4$ (available at www.jneurosci.org as supplemental material).

brain function, and behaviors, including the sleep-wake cycle and vice versa.

\section{Materials and Methods}

Infants. Fifty-two full-term healthy Japanese infants participated in the present study. Fifteen infants were neonates ( 6 girls and 9 boys; mean age, $4.3 \mathrm{~d}$; range, $2-11 \mathrm{~d}$ ), 21 were 3 months old (11 girls and 10 boys; mean age, $111.6 \mathrm{~d}$; range, $102-123 \mathrm{~d}$ ), and 16 were 6 months old ( 6 girls and 10 boys; mean age, $197.0 \mathrm{~d}$; range, 180-206 d). All infants were sleeping naturally while they were studied. The infants were almost motionless and slept soundly throughout the measurements. Slow movements, burst of activity, and non-nutritive sucking were observed less than three times in total in the measurement of each infant. Informed consent was obtained from the parents of the infants before the initiation of the experiments. The study was approved by the ethics committees of Tokyo Women's Medical University for studying the neonates and Graduate School of Education, University of Tokyo for studying the 3- and 6-month-old infants.

NIRS recordings. We used multichannel NIRS instruments (ETG7000; Hitachi Medical Corporation) in this study. NIRS exploits the 
A

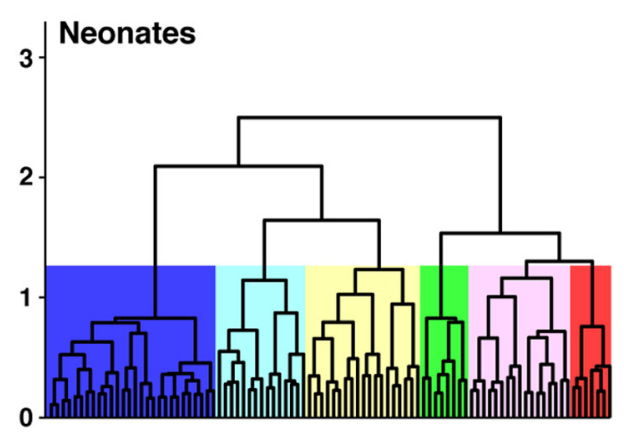

D

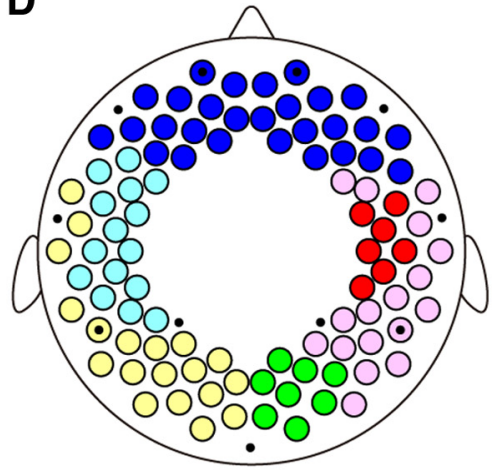

B

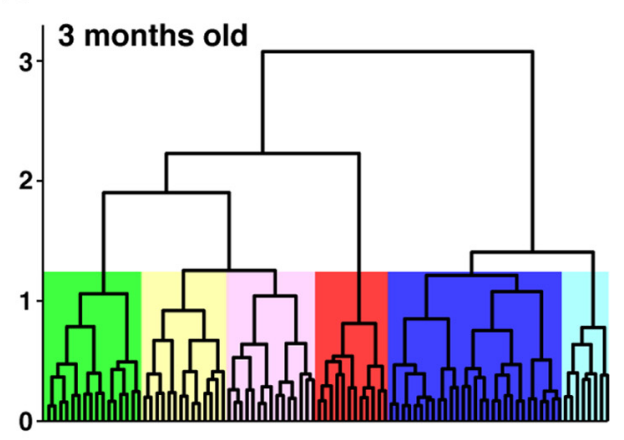

C

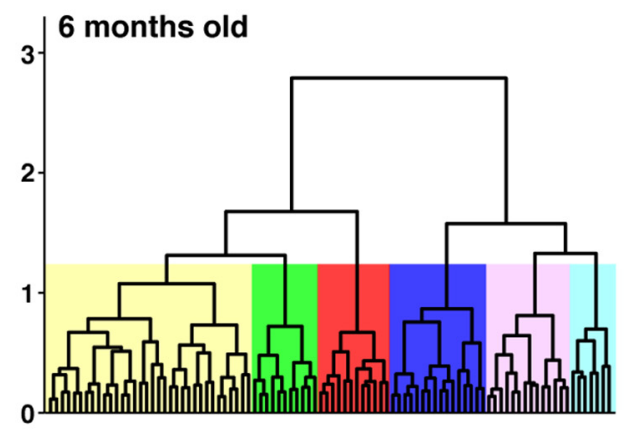

E

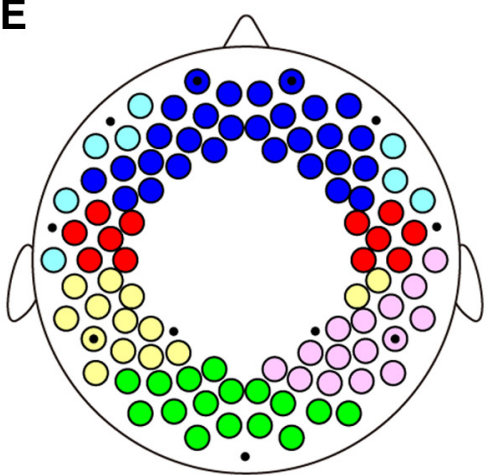

$\mathbf{F}$

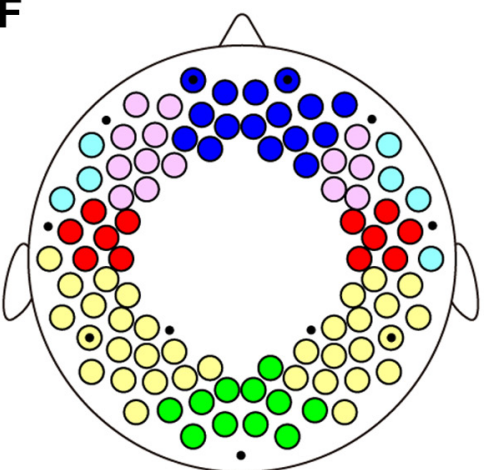

Figure 2. Clusters divided according to the temporal correlations. $\boldsymbol{A}-\boldsymbol{C}$, We defined the distance between two channels by calculating $1-r$ and conducted cluster analyses. Each leaf in the dendrogram corresponds to a measurement channel. The six clusters in each infant group can be identified by coloring. $\mathbf{D}-\boldsymbol{F}$, Spatial configuration of six clusters obtained by cluster analyses. The small dots are the landmarks shown in Figure $1 A$ (red dots).

optical properties of hemoglobin, which has oxygenated (oxy-Hb) and deoxygenated (deoxy-Hb) forms with different absorption spectra in the near-infrared (NIR) wavelength region. By using two NIR wavelengths $(785$ and $830 \mathrm{~nm})$ and analyzing data on the basis of modified LambertBeer law, these instruments measure the relative changes in the concentrations of oxy-Hb and deoxy-Hb in the cerebral cortex at preset measurement regions, with a $0.1 \mathrm{~s}$ time resolution. Because of this sampling rate of NIRS measurements, effects of systemic physiology, such as respiration and cardiovascular oscillations, are unlikely to be aliased into lower-frequency bands. Detailed descriptions of the principles underlying NIRS have been described previously (Jöbsis, 1977; Reynolds et al., 1988; Maki et al., 1995; Villringer and Chance, 1997; Obrig and Villringer, 2003).

We previously revealed the patterns of spontaneous oscillation in the infant brain by using multichannel NIRS (Taga et al., 2000). NIRS has also been successfully used to investigate cortical activation in infants (Peña et al., 2003; Taga et al., 2003; Kotilahti et al., 2005; Homae et al., 2006, 2007; Minagawa-Kawai et al., 2007; Taga and Asakawa, 2007; Taga et al., 2007; Nakano et al., 2008, 2009; Watanabe et al., 2008). NIR light was emitted from laser diodes through incident optical fibers (maximum intensity: $0.4 \mathrm{~mW}$ for neonates, $0.6 \mathrm{~mW}$ for 3-month-old infants, and $1.2 \mathrm{~mW}$ for 6 -month-old infants). The received light was detected by avalanche photodiodes via optical fibers and separated into individual light sources, depending on each wavelength.

We used two sets of $3 \times 10$ arrays composed of 15 incident and 15 detection fibers mounted on a flexible cap over the frontal, temporal, parietal, and occipital areas of each hemisphere (Fig. 1A). A pair of adjacent incident and detection fibers formed a single measurement channel (a total of 47 channels for each $3 \times 10$ array); this enabled us to simultaneously measure the time course of oxy- $\mathrm{Hb}$ and deoxy- $\mathrm{Hb}$ signals. The distance between the incident and detection fibers was set at $\sim 2 \mathrm{~cm}$ (Taga et al., 2007). The measurement channels were correctly positioned by referring to the international 10-20 system of electrode placement and using the external auditory pores, vertex, and inion as landmarks in each infant. Because only a few atlases of the infant brain are available, we used previous studies on adults (Homan et al., 1987; Steinmetz et al., 1989; Herwig et al., 2003; Okamoto et al., 2004) to estimate the craniocerebral correlation for each measurement channel. We have reported previously a functional map of audiovisual stimuli in infants, which is consistent with an estimated map from the craniocerebral correlation (Watanabe et al., 2008).

Data analysis. In a previous study on infants, we found that oxy-Hb signals had a better signal-to-noise ratio than deoxy-Hb signals (Homae et al., 2007), and therefore, we examined the variation in the oxy- $\mathrm{Hb}$ signals to estimate the changes in regional cerebral blood oxygenation during brain activation. We evaluated the relative changes in oxy- $\mathrm{Hb}$ signals contingent on an arbitrarily assigned zero baseline from the start of the measurement period, which was based on the modified LambertBeer law. Because the precise optical path length of the light traveling through brain tissue cannot be determined, the unit for the oxy-Hb signals was calculated by multiplying the molar concentration by length (millimolar $\times$ millimeter $)$.

For each individual dataset, we used a bandpass filter from 0.005 to 1 $\mathrm{Hz}$ and extracted $3 \mathrm{~min}$ data (i.e., 1800 time points) from the continuous time courses. The bandpass filter eliminated cardiac pulsation $(\sim 2 \mathrm{~Hz}$ in infants) and smoothed signal drifts over long timescales and motion artifacts. To confirm our results using this bandpass filter (from 0.005 to $1 \mathrm{~Hz}$ ), we also used a narrower bandpass filter from 0.009 to $0.08 \mathrm{~Hz}$, which has been used in previous studies to eliminate cardiac and respiratory rhythms (Fox et al., 2005; White et al., 2009). These filters were in line with the purpose of our present study if we could obtain consistent analysis results using two types of bandpass filters.

We analyzed the power spectrum of the continuous data obtained from each infant group to examine time-series properties. For each infant data, we calculated the correlation coefficients $(r)$ between the time course of a single channel and the time course from all other measurement channels [number of pairs: $(94 \times 93) / 2=4371$ ]. The 3 min data of two channels determined a single $r$ value. We used all the filtered data to estimate the cross-correlations. Because we used correlation coefficients in our analyses, the different optical path lengths of the measurement 

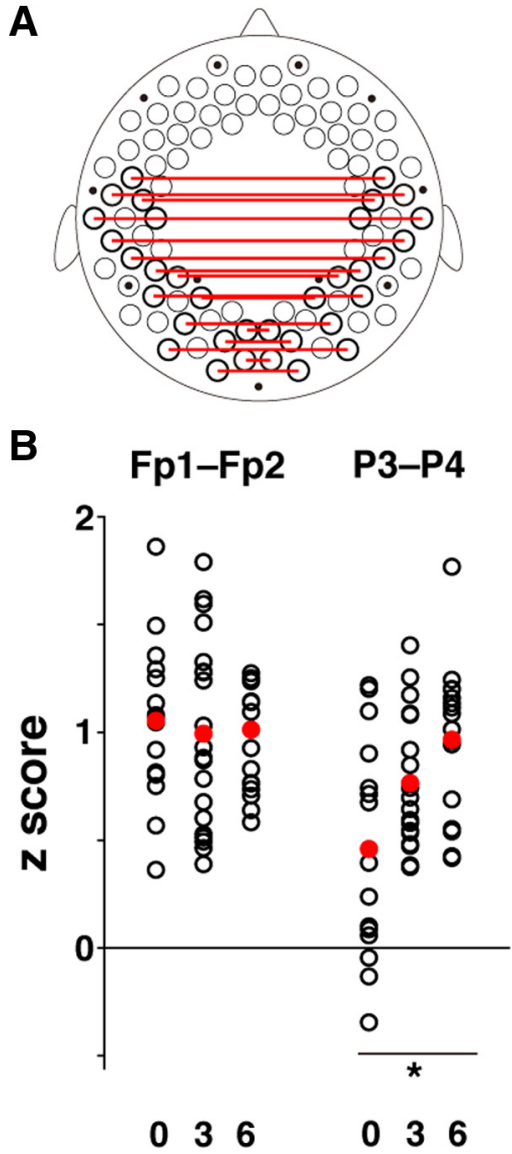

Figure 3. The developmental changes in correlations between the homologous channels. $\boldsymbol{A}$, The red lines connect channels ( 17 of 47 channel pairs) that showed significant changes, as revealed by ANOVA ( $p<0.05$, corrected). $\boldsymbol{B}$, Individual data of temporal correlations normalized to $z$ scores. The scatter plots show $z$ scores in frontal (Fp1-Fp2) and parietal (P3-P4) pairs. The red circles indicate mean values in each infant group. The differences between the infant groups are shown ( ${ }^{*} p<0.05$, post hoc test, Tukey's HSD).

channels did not affect our results. We considered both positive and negative $r$ values (range, $-1 \leq r \leq 1$ ) and evaluated all the $r$ values.

In the cluster analyses, we defined the distance between the two channels by calculating $1-r$. We applied the Ward method for determining the distance and constructed a dendrogram. We showed six clusters for each infant group.

To reveal the developmental changes in connectivity, we analyzed the differences among the infant groups on a channel pair basis. We first converted the $r$ values to $z$ scores by Fischer's $z$ transformation. We evaluated the individual $z$ scores as random effects and performed ANOVA with subject groups as a factor (three groups of 52 infants in total). Because we assumed that the cross-correlation between every pair of channels was independent of that calculated in the other pairs, multiple comparisons among the pairs of channels were considered by adopting a measurement-channels false discovery rate correction at $q<0.05$ (Benjamini and Hochberg, 1995; Genovese et al., 2002; Singh and Dan, 2006). Tukey's honestly significant difference (HSD) tests were applied as post hoc tests.

\section{Results}

We set up 94 measurement channels over the frontal, temporal, parietal, and occipital regions of neonates, 3-month-old infants, and 6-month-old infants (Fig. $1 \mathrm{~A}$ ). All measurements of spontaneous fluctuations were taken when the infants were in sleep. The obtained signals showed complex time-series properties, which were characterized as $1 / f$ fluctuations (supplemental Fig. 1, available at www.jneurosci.org as supplemental material). To reveal

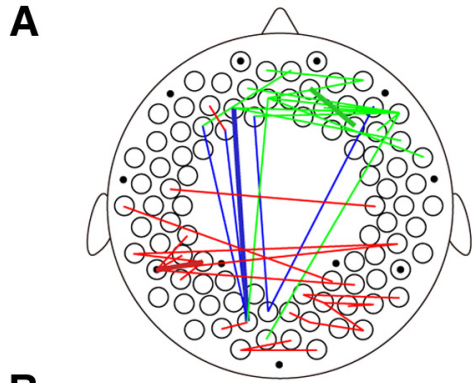

B

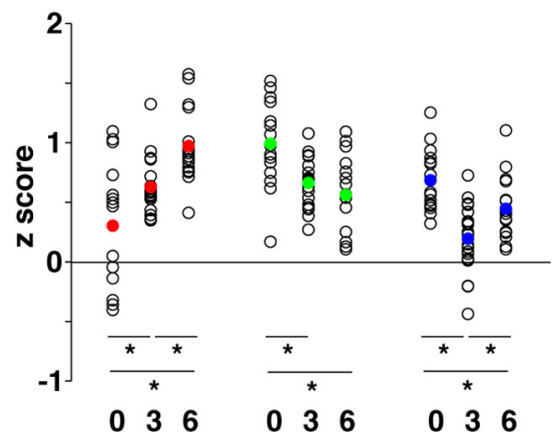

Figure 4. Increase, decrease, and U-shaped changes in connectivity during the course of development. $\boldsymbol{A}$, The red, green, and blue lines connect channels that showed significant changes in temporal correlations, as revealed by ANOVAs and post hoc tests (red: neonates $<3$ and 6-month-old infants; green: neonates $>3$ - and 6-month-old infants; blue: neonates and 6-month-old infants $>3$-month-old infants). $\boldsymbol{B}$, Individual data of correlations normalized to $\boldsymbol{Z}$ scores. The data for connections shown by bold lines in $\boldsymbol{A}$ are presented. The differences between the infant groups are shown ( ${ }^{*} p<0.05$, post hoc tests, Tukey's HSD).

the functional connectivity of the cortical networks, we calculated the temporal correlations of continuous signals between all pairs of measurement channels for each infant (Fig. 1B). Although the distribution of correlations in neonates was left skewed, that in the 3- and 6-month-old infants was almost symmetrical around the median values (Fig. 1C) (KolmogorovSmirnov tests, $p<0.001$ for all pairs of groups). We found that the frontal region of neonates showed high correlations across multiple channels within and between hemispheres (Fig. 1D) (supplemental Fig. 2, available at www.jneurosci.org as supplemental material). Notable interhemispheric correlations were observed in the 3- and 6-month-old infants (Fig. 1D) (supplemental Figs. 3, 4, available at www.jneurosci.org as supplemental material).

To reveal the spatial formation of regions that had similar temporal correlations, we applied cluster analyses to the correlation coefficients (Fig. 2A-C). In the neonates, we found that the frontal region formed a tight cluster over the midline (Fig. $2 D$ ). The adjacent or closely located channels in the temporal, parietal, and occipital regions formed clusters within a hemisphere. In the 3- and 6-month-old infants, in contrast, clusters were formed across the midline, which included homologous channels (Fig. $2 E, F$ ). In the 3-month-old infants, the clusters above T3 and T4 (Fig. 2 E, red cluster) showed notable consistency in terms of spatial configuration in both the hemispheres. This pattern was replicated in the 6-month-old infants (Fig. 2 F), thus establishing the validity of the analysis. These clusters were observed even if we used the narrower bandpass filter (supplemental Fig. 5, available at www.jneurosci.org as supplemental material). Our findings suggest that the bilateral organization of spontaneous networks begins to emerge during the first 3 months of life. 
The connectivity between homologous regions in the left and right hemispheres characterizes bilateral cerebral organization. Developmental changes in the correlations over the midline of the brain were assessed using statistical analyses (one-way ANOVA, $p<0.05$, corrected using the false-discovery rate among 47 pairs); significant increases were observed in the posterior regions but not in the frontal region (Fig. 3). We further calculated the magnitudes of squared coherence between the homologous regions. A coherence value below $0.1 \mathrm{~Hz}$ was high and reached a plateau in all three infant groups, indicating that the lower frequencies are dominant across the $1 / f$ band (supplemental Fig. 6, available at www.jneurosci.org as supplemental material). As in the correlations, squared coherences below $0.1 \mathrm{~Hz}$ increased during the course of development. The increases in the interhemispheric correlations, which are in line with the high correlations between homologous regions observed in adult studies (Biswal et al., 1995; Salvador et al., 2005; Buckner and Vincent, 2007; Fox and Raichle, 2007; Seeley et al., 2009; White et al., 2009), are expected to be related to the functional maturation of the corpus callosum.

The parietal regions of the 6-month-old infants had correlations with channels not only in the contralateral hemisphere (Fig. 3) but also in the ipsilateral hemisphere (Fig. 1C). We found that the channels in the left parietal (near P3) and temporal (T5) regions showed developmental increases in correlation (Fig. 4A, red bold line, $B$, left $)\left(F_{(2,49)}=13.1, p<0.00005\right.$; post hoc tests, $p<0.05$ ). In contrast, the homologous right pair (P4 and T6) did not show any significant changes $\left(F_{(2,49)}=1.7, p>0.1\right)$. The left-lateralized development of functional connectivity is consistent with the leftward asymmetry of the arcuate fasciculus in adult humans (Rilling et al., 2008), suggesting that long-range connectivity, as a basis for language acquisition, occurs in early infancy.

Although developmental increases in correlations were mainly observed in the temporal and posterior regions (Fig. 4A, red lines), we found that pairs of frontal channels showed developmental decreases in correlations (Fig. $4 A$, green lines, $B$, middle) $\left(F_{(2,49)}=9.3, p<0.0005\right)$. The channel pairs showing increases and those showing decreases in correlations were not randomly intermixed in the whole brain but were isolated depending on their location in the cortical regions. Interestingly, we found channel pairs that exhibited nonmonotonic changes in correlations. The correlations between several channels in the frontal and occipital regions showed significant decreases from the neonatal period to the age of 3 months and significant increases from the age of 3 months to the age of 6 months (Fig. $4 A$, blue lines, $B$, right $)\left(F_{(2,49)}=14.7, p<0.00005\right)$. The channels that showed this U-shaped pattern were mostly lateralized to the left hemisphere. Future studies should examine how the three types of cortical networks are modulated when sensory stimuli are presented to infants.

\section{Discussion}

In the present study, we revealed that, in infants, the organization of cortical networks with regard to increase and/or decrease in functional connectivity depends on the cortical region. The developmental changes involved in the organization of cortical networks are classified into the three following patterns: connectivity increases in the temporal and posterior regions, decreases in the frontal region, and U-shaped changes occur in the frontoposterior region. The increases in the correlations reflect the strengthening of the relationship between cortical regions, which might be the basis of constructive organization in the developing brain (Quartz and Sejnowski, 1997; Sirois et al., 2008). In contrast, the decreases in correlations reflect pruning of connections between cortical regions. The decreases in the regional relationships may be related to selective organization of the postnatal brain (Changeux and Dehaene, 1989) after the elimination of superfluous connections. The interpretation of the U-shaped changes is not straightforward, but we think that the U-shaped pattern may represent the reorganization of connectivity between the frontal and posterior regions. For example, strong correlations between the two regions in neonates reflect synchronized activation of thalamocortical networks, and their resonance becomes weaker at the age of 3 months because of pruning or elimination of thalamocortical connections. Furthermore, corticocortical connections increase at the age of 6 months, resulting in strong correlations among the long-range networks. This reorganization may be caused by developmental changes within corticocortical networks or within thalamocortical networks. Behavioral studies have reported U-shaped development in infancy (Muir et al., 1989; Morton and Johnson, 1991); exploration of $\mathrm{U}$-shaped changes in the infant brain would bridge the gap between behavioral and neuroscience studies on early human development.

We elucidated the dynamics of the organization of cortical networks by analyzing the spontaneous fluctuations in the brain activity of infants. The spontaneous fluctuations extend over wide frequency bands, whereas both the mechanisms and contributions of the lowest frequencies to spatially specific correlations have not been systematically investigated (Fox and Raichle, 2007). Our results of the coherence analysis and correlation analyses by using two types of bandpass filter (lowest cutoff, 0.005 and $0.009 \mathrm{~Hz}$ ) demonstrated that the correlations between two regions were not solely determined by the lowest frequencies. The roles played by the spontaneous fluctuations in the construction of multiple functional networks in the brain, including the default-mode network (Fransson et al., 2007; Gao et al., 2009) and networks for perception and cognition (Imada et al., 2006; Homae et al., 2007; Watanabe et al., 2008; Nakano et al., 2009), are very interesting. Our results suggest that emerging global networks, manifested as long-range connectivity within and between hemispheres, constrain functional brain development. Our methods can be used to detect disorganized formation of global networks in developmental disorders such as autism (Courchesne et al., 2007), although physiological confounds, including respiratory and cardiovascular oscillations, should be carefully considered. Network analysis of the infant brain would help provide a deep understanding of brain organization, cognitive development in infancy, and the origin of human nature.

\section{References}

Benjamini Y, Hochberg Y (1995) Controlling the false discovery rate: a practical and powerful approach to multiple testing. J R Stat Soc Ser B Stat Methodol 57:289-300.

Biswal B, Yetkin FZ, Haughton VM, Hyde JS (1995) Functional connectivity in the motor cortex of resting human brain using echo-planar MRI. Magn Reson Med 34:537-541.

Buckner RL, Vincent JL (2007) Unrest at rest: default activity and spontaneous network correlations. Neuroimage 37:1091-1099; discussion 1097-1099.

Changeux JP, Dehaene S (1989) Neuronal models of cognitive functions. Cognition 33:63-109.

Courchesne E, Pierce K, Schumann CM, Redcay E, Buckwalter JA, Kennedy DP, Morgan J (2007) Mapping early brain development in autism. Neuron 56:399-413.

Dehaene-Lambertz G, Dehaene S, Hertz-Pannier L (2002) Functional neuroimaging of speech perception in infants. Science 298:2013-2015.

Dubois J, Dehaene-Lambertz G, Perrin M, Mangin JF, Cointepas Y, Duchesnay E, 
Le Bihan D, Hertz-Pannier L (2008) Asynchrony of the early maturation of white matter bundles in healthy infants: quantitative landmarks revealed noninvasively by diffusion tensor imaging. Hum Brain Mapp 29:14-27.

Fox MD, Raichle ME (2007) Spontaneous fluctuations in brain activity observed with functional magnetic resonance imaging. Nat Rev Neurosci 8:700-711.

Fox MD, Snyder AZ, Vincent JL, Corbetta M, Van Essen DC, Raichle ME (2005) The human brain is intrinsically organized into dynamic, anticorrelated functional networks. Proc Natl Acad Sci U S A 102:9673-9678.

Fransson P, Skiöld B, Horsch S, Nordell A, Blennow M, Lagercrantz H, Aden U (2007) Resting-state networks in the infant brain. Proc Natl Acad Sci U S A 104:15531-15536.

Gao W, Zhu H, Giovanello KS, Smith JK, Shen D, Gilmore JH, Lin W (2009) Evidence on the emergence of the brain's default network from 2-weekold to 2-year-old healthy pediatric subjects. Proc Natl Acad Sci U S A 106:6790-6795.

Genovese CR, Lazar NA, Nichols T (2002) Thresholding of statistical maps in functional neuroimaging using the false discovery rate. Neuroimage 15:870-878.

Hermoye L, Saint-Martin C, Cosnard G, Lee SK, Kim J, Nassogne MC, Menten R, Clapuyt P, Donohue PK, Hua K, Wakana S, Jiang H, van Zijl PC, Mori S (2006) Pediatric diffusion tensor imaging: normal database and observation of the white matter maturation in early childhood. Neuroimage 29:493-504.

Herwig U, Satrapi P, Schönfeldt-Lecuona C (2003) Using the International 10-20 EEG system for positioning of transcranial magnetic stimulation. Brain Topogr 16:95-99.

Homae F, Watanabe H, Nakano T, Asakawa K, Taga G (2006) The right hemisphere of sleeping infant perceives sentential prosody. Neurosci Res 54:276-280.

Homae F, Watanabe H, Nakano T, Taga G (2007) Prosodic processing in the developing brain. Neurosci Res 59:29-39.

Homan RW, Herman J, Purdy P (1987) Cerebral location of international 10-20 system electrode placement. Electroencephalogr Clin Neurophysiol 66:376-382.

Huttenlocher PR (2002) Neural plasticity: the effects of environment on the development of the cerebral cortex. Cambridge, MA: Harvard UP.

Imada T, Zhang Y, Cheour M, Taulu S, Ahonen A, Kuhl PK (2006) Infant speech perception activates Broca's area: a developmental magnetoencephalography study. Neuroreport 17:957-962.

Jöbsis FF (1977) Noninvasive, infrared monitoring of cerebral and myocardial oxygen sufficiency and circulatory parameters. Science 198:1264-1267.

Johnson MK (2005) Developmental cognitive neuroscience, Ed 2. Malden, MA: Blackwell.

Kotilahti K, Nissilä I, Huotilainen M, Mäkelä R, Gavrielides N, Noponen T, Björkman P, Fellman V, Katila T (2005) Bilateral hemodynamic responses to auditory stimulation in newborn infants. Neuroreport 16:13731377.

LaMantia AS, Rakic P (1990) Axon overproduction and elimination in the corpus callosum of the developing rhesus monkey. J Neurosci 10:2156-2175.

Maki A, Yamashita Y, Ito Y, Watanabe E, Mayanagi Y, Koizumi H (1995) Spatial and temporal analysis of human motor activity using noninvasive NIR topography. Med Phys 22:1997-2005.

Minagawa-Kawai Y, Mori K, Naoi N, Kojima S (2007) Neural attunement processes in infants during the acquisition of a language-specific phonemic contrast. J Neurosci 27:315-321.

Morton J, Johnson MH (1991) CONSPEC and CONLERN: a two-process theory of infant face recognition. Psychol Rev 98:164-181.

Muir DW, Clifton RK, Clarkson MG (1989) The development of a human auditory localization response: a U-shaped function. Can J Psychol 43:199-216.
Nakano T, Homae F, Watanabe H, Taga G (2008) Anticipatory cortical activation precedes auditory events in sleeping infants. PLoS One 3:e3912.

Nakano T, Watanabe H, Homae F, Taga G (2009) Prefrontal cortical involvement in young infants' analysis of novelty. Cereb Cortex 19:455-463.

Obrig H, Villringer A (2003) Beyond the visible: imaging the human brain with light. J Cereb Blood Flow Metab 23:1-18.

Okamoto M, Dan H, Sakamoto K, Takeo K, Shimizu K, Kohno S, Oda I, Isobe S, Suzuki T, Kohyama K, Dan I (2004) Three-dimensional probabilistic anatomical cranio-cerebral correlation via the international 10-20 system oriented for transcranial functional brain mapping. Neuroimage 21:99-111.

Paus T, Collins DL, Evans AC, Leonard G, Pike B, Zijdenbos A (2001) Maturation of white matter in the human brain: a review of magnetic resonance studies. Brain Res Bull 54:255-266.

Peña M, Maki A, Kovacić D, Dehaene-Lambertz G, Koizumi H, Bouquet F, Mehler J (2003) Sounds and silence: an optical topography study of language recognition at birth. Proc Natl Acad Sci U S A 100:11702-11705.

Quartz SR, Sejnowski TJ (1997) The neural basis of cognitive development: a constructivist manifesto. Behav Brain Sci 20:537-556; discussion 556-596.

Reynolds EO, Wyatt JS, Azzopardi D, Delpy DT, Cady EB, Cope M, Wray S (1988) New non-invasive methods for assessing brain oxygenation and hemodynamics. Br Med Bull 44:1052-1075.

Rilling JK, Glasser MF, Preuss TM, Ma X, Zhao T, Hu X, Behrens TE (2008) The evolution of the arcuate fasciculus revealed with comparative DTI. Nat Neurosci 11:426-428.

Salvador R, Suckling J, Coleman MR, Pickard JD, Menon D, Bullmore E (2005) Neurophysiological architecture of functional magnetic resonance images of human brain. Cereb Cortex 15:1332-1342.

Seeley WW, Crawford RK, Zhou J, Miller BL, Greicius MD (2009) Neurodegenerative diseases target large-scale human brain networks. Neuron 62:42-52.

Singh AK, Dan I (2006) Exploring the false discovery rate in multichannel NIRS. Neuroimage 33:542-549.

Sirois S, Spratling M, Thomas MS, Westermann G, Mareschal D, Johnson MH (2008) Precis of neuroconstructivism: how the brain constructs cognition. Behav Brain Sci 31:321-331; discussion 331-356.

Steinmetz H, Fürst G, Meyer BU (1989) Craniocerebral topography within the international 10-20 system. Electroencephalogr Clin Neurophysiol 72:499-506.

Taga G, Asakawa K (2007) Selectivity and localization of cortical response to auditory and visual stimulation in awake infants aged 2 to 4 months. Neuroimage 36:1246-1252.

Taga G, Konishi Y, Maki A, Tachibana T, Fujiwara M, Koizumi H (2000) Spontaneous oscillation of oxy- and deoxy-hemoglobin changes with a phase difference throughout the occipital cortex of newborn infants observed using non-invasive optical topography. Neurosci Lett 282:101-104.

Taga G, Asakawa K, Maki A, Konishi Y, Koizumi H (2003) Brain imaging in awake infants by near-infrared optical topography. Proc Natl Acad Sci U S A 100:10722-10727.

Taga G, Homae F, Watanabe H (2007) Effects of source-detector distance of near infrared spectroscopy on the measurement of the cortical hemodynamic response in infants. Neuroimage 38:452-460.

Villringer A, Chance B (1997) Non-invasive optical spectroscopy and imaging of human brain function. Trends Neurosci 20:435-442.

Watanabe H, Homae F, Nakano T, Taga G (2008) Functional activation in diverse regions of the developing brain of human infants. Neuroimage 43:346-357.

White BR, Snyder AZ, Cohen AL, Petersen SE, Raichle ME, Schlaggar BL, Culver JP (2009) Resting-state functional connectivity in the human brain revealed with diffuse optical tomography. Neuroimage 47:148-156. 